\title{
Dermal papillae flattening of thigh skin in Conus Cauda Syndrome
}

\author{
Barbara Ravara (1,2), Christian Hofer (3), Helmut Kern (3,4); Diego Guidolin (5), \\ Andrea Porzionato (5), Raffaele De Caro (5); Giovanna Albertin (5)
}

(1) Interdepartmental Research Center of Myology, Department of Biomedical Science, University of Padova, Italy; (2) A\&C M-C Foundation for Translational Myology, Padova, Italy; (3) Ludwig Boltzmann Institute of Electrical Stimulation and Physical Rehabilitation, Vienna, Austria; (4) Physiko- und Rheumatherapie, St. Poelten, Austria; (5) Interdepartmental Research Center of Myology, Department of Neuroscience, Section of Human Anatomy, University of Padova, Italy

This article is distributed under the terms of the Creative Commons Attribution Noncommercial License (CC BY-NC 4.0) which permits any noncommercial use, distribution, and reproduction in any medium, provided the original author(s) and source are credited.

\begin{abstract}
Our previous studies have shown that severely atrophic Quadriceps muscles of spinal cord injury (SCI) persons suffering with complete conus and cauda equina syndrome, and thus with permanent denervation-induced atrophy and degeneration of muscle, were almost completely rescued to normal size after two years of home based Functional Electrical Stimulation (hbFES). Since large surface electrodes were used to stimulate the denervated thigh muscles, we wanted to know if the skin was affected by this peculiar long-term treatment. Indeed, we demonstrated by two approaches that the epidermis decreases in thickness in the long term denervated persons, while it increased to almost pre-SCI values in hbFES compliant SCI persons. Here we report data of morphometry of skin biopsies from both legs of 18 SCI persons, harvested at enrolment in the Project RISE, to test if the Interdigitation Index, a simple measurement of the epidermaldermal junction, may provide a further precise quantitative evidence of the flattening of the skin in those SCI persons. The Interdigitation Index of the 36 skin biopsies shows a higly significant linear correlation with the years of SCI $(\mathrm{p}<0.001)$. Furthermore, when the 18 SCI persons are divided in two groups ( 1 to 3.9 versus 4.1 to 8.0 years from SCI, respectively) and the data are compared, the later Group presents a statistically significant $-22 \%$ decrease (p, 0.029) of the Interdigitation Index. On the other hand counting the papille do not provide the same strong evidence. In conclusion, the Interdigitation Index is an additional sound quantitative structural biomarker of skin atrophy and flattening occurring in SCI. The result correlates with the much severe extent of atrophy of the permanently denervated thigh muscles, as determined at both macro and microscopic levels. We are confident that the Interdigitation Index will provide sound evidence that the effects of hbFES, we previously reported on skeletal muscle and epidermis thickness, will be extended to the dermal layer of the skin, suggesting a coordinated negative effects of SCI on skeletal muscle and skin, and an improvement of both tissues after hbFES. Incoming analyses will be extended to basal lamina, collagene types, elastic fibers and skin annexes in the subcutaneous layer.
\end{abstract}

Key Words: spinal cord injury, denervated-degenerating muscle, h-bFES induced recovery, skin biopsy, Skin morphometry, Interdigitation Index

Atrophic leg muscles from spinal cord injury patients suffering with complete conus and cauda equina lesions, and thus with permanent-denervation of thigh muscles, are almost completely rescued to almost normal size and tetanic contractility after two years of home-based Functional Electrical Stimulation (hbFES). This impressive improvements of the leg structure and functions of paraplegics with long-term denervated degenerating muscles (DDM) were supported by strong evidence collected during and at the end of the EU RISE Program [Use of electrical stimulation to restore standing in paraplegics with long-term DDMs (QLG5-CT-200102191)]. ${ }^{1-13}$ Indeed we reported sound data of FESinduced muscle contraction strength, muscle bulk and 


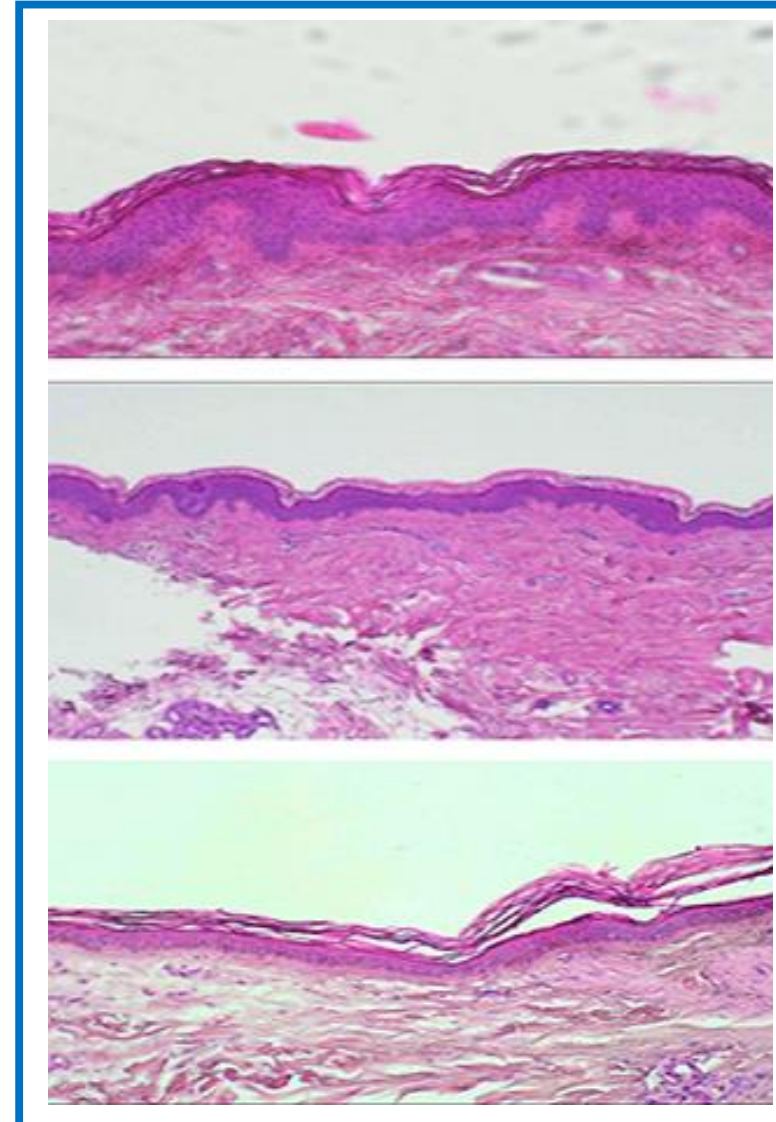

Fig 1. Skin biopsy from SCI persons after less than 1 (upper panel), at 3 (middle panel), and at 8 (lower panel) years from SCI. The Interdigitation Index is quantified by ImageJ measuring the dermal to epidermis profile versus the length of the image $(1 \mathrm{~mm})$. The number of the dermal papillae was also counted in each image.

muscle fibers structural characteristics at both microscopic and ultra microscopic observation., , $3,5-8,10^{-}$ Since we used a purpose designed electro stimulator and large surface electrodes, both now commercialyy available, ${ }^{14}$ to stimulate the thigh muscles, we wanted to know if the electrostimulated skin was affected. To evaluate skin characteristics we analyzed skin biopsies harvested from a subsets of patients enrolled in the EU Project RISE. ${ }^{2}$ Morphometric analyses of the thickness and area of epidermis determined in skin cross sections before and after two years of hbFES demonstrated that those parameters were negatively correlated with the years from SCI and, importantly, recovered to almost normal values after two years of hbFES, despite the fact that the values were observed after two additional years of permanent denervation. ${ }^{15,16}$

Here we report morphometry data of 36 skin biopsies (harvested from both legs of 18 SCI persons at time of enrolment in the Project RISE) to test if the Interdigitation Index, a simple measurement of the epidermal-dermal junction, ${ }^{17}$ may provide a further precise quantitative evidence of the flattening of the skin in those SCI persons.

\section{Materials and Methods \\ Histologic morphometry of skin biopsies}

The harvested skin biopsies were fixed in $10 \%$ buffered formalin and embedded in paraffin. Histological sections of $5 \mu \mathrm{m}$ thickness were then collected and stained standard Ematoxilin-Eosin stain. Three sections per sample were considered for the analysis and from each transverse section 3-5 digital images were collected at a primary magnification of x10 using a light microscope (B-293 PLi, Optika Srl, Ponteranica, BG, Italy) mounting a HDMI camera (Optika 4083.13E HDMI Easy camera). Image of $1024 \times 576$ pixel size were then captured and stored as TIFF files on the workstation associated with the instrument. Care was taken to accurately align the epidermal layer parallel to the largest side of the frame before acquiring each image. Images were analyzed by using the ImageJ software (available at http://rsb.info.nih.gov/ij/). ${ }^{18}$ The size of the captured images was on the order of $1024 \times 576$ pixels and was converted to $\mu \mathrm{m}$. The thickness of the epidermis was evaluated on three fields per section of acquired images (Fig. 1). A grid was used on the traced images and 1 at least 10 different measurements were performed using ImageJ. A series of skin sections were also used to immunhistochemically determine the presence of Langherhans cells. The boundary of the epidermal layer, from the outermost surface of the epidermis (but excluding the stratum corneum) to the dermo-epidermal junction, was interactively traced to estimate its area. ${ }^{18}$ All the sections were finally counterstained with hematoxylin (EnVision ${ }^{\mathrm{TM}}$ FLEX Hematoxylin, Dako) for 5 min to reveal the presence of nuclei, dehydrated with a increasing scale of alcohol solutions $(70 \%, 95 \%, 99 \%)$, cleared with xylene and mounted. The Interdigitation Index was quantified by ImageJ measuring the dermal to epidermis profile versus the length of the image $(1 \mathrm{~mm})$. The numbers of the dermal papillae were also counted in each image and expressed per $1 \mathrm{~mm}$ of basal lamina length in each image.

\section{Statistical analyses}

Statistical analyses were performed by using GraphPad Prism 5.0 software (GraphPad software, La Jolla, CA, USA). The limit for statistical significance was always considered $\mathrm{p}<0.05$.

\section{Results}

The thirtysix skin biopsies here analyzed were harvested, a few weeks before hbFES, from both thighs of 18 SCI patients enrolled in the EU Program RISE [Use of electrical stimulation to restore standing in paraplegics with long-term DDMs (QLG5-CT-2001-02191)]. ${ }^{1-8}$ In $\mathrm{H}-\mathrm{E}$ staned transverse section of the skin, all biopsies presented with a regular multilayer squamous epithelium 


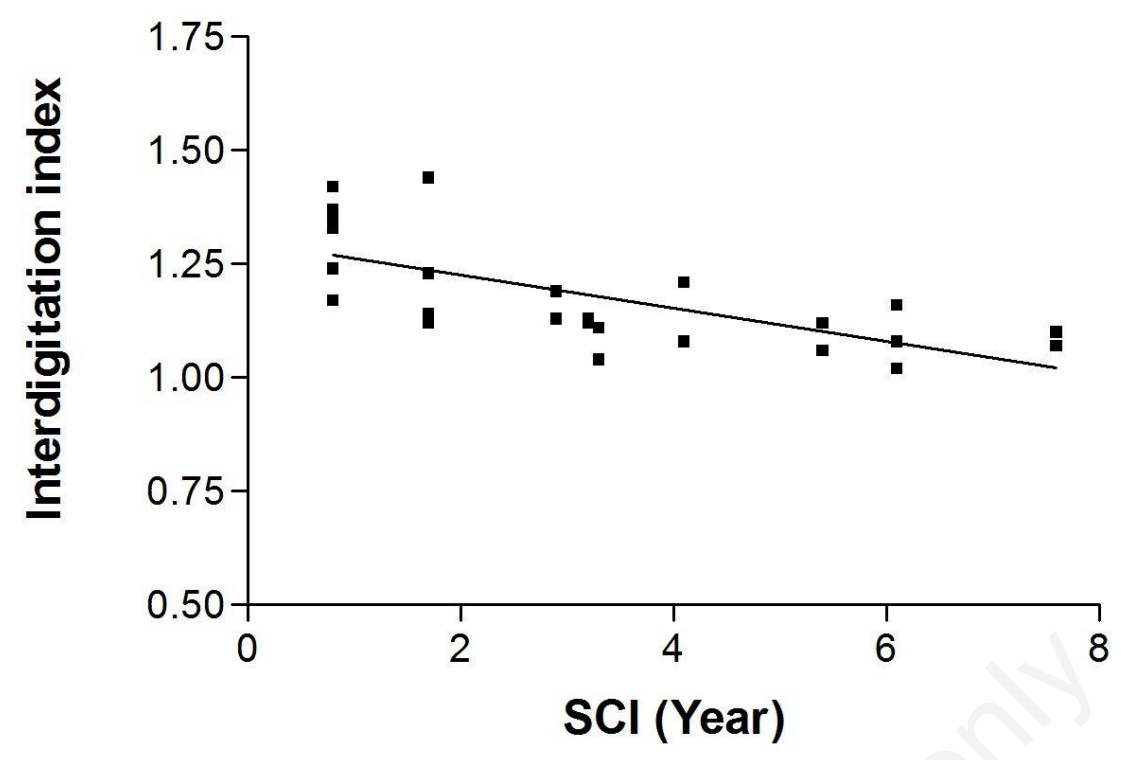

Fig 2. Linear regression of values of the Interdigitation Index of the skin biopsies harvested at enrollment of the RISE Trial in between 1 and 8 Years from SCI. $R^{2}=0.485, p<0.001$.

rich in cells. The epidermis was always distinguishable from the underlying dermis and exhibited a regular basal membrane. The $\mathrm{H}-\mathrm{E}$ staining revealed the various layers of keratinocytes with small, nucleated basal cells. ${ }^{15}$. The surface layer is the stratum corneum which sloughs away naturally and is evenly colored, thus it was not included in the measured cross area of the epidermis. Although some samples shown the skin organization as having ridges and papillae, in several samples the epidermis appears thinner and flattened, in particular in the skin biopsies harvested before h-bFES, from 3 to 8 years after SCI. We previously performed skin morphometry, measuring both thickness, ${ }^{15}$ and the total area of epidermis, ${ }^{16}$ in transverse sections of skin biopsies, by acquiring ImageJ pictures. Here we report the results of the Interdigitation Index and the numbers of papillae per $1 \mathrm{~mm}$ of skin in 36 biopsies harvetsted from SCI persons at the enrolment stage, i.e., before hbFES was started. Despite the heterogeneity of gender and time from SCI of these 18 cases, ${ }^{1-3}$ the results in Fig. 2 show that the Interdigitation Index of the 36 skin biopsies shows a higly significant linear correlation with the years from SCI $(\mathrm{p}<0.001)$. Furthermore, Table 1 confirms that when the 18 SCI persons are divided in two groups (1 to 3.9 versus 4.1 to 8.0 years from SCI, respectively) and the data are compared, the later Group presents a statistically significant $-22 \%$ decrease $(p=0.029)$ of the Interdigitation Index, while counting the papille do not provide the same strong quantitative evidence of the skin atrophy and flattening process (Table 1).

Table 1. Interdigitation Index and number of papillae per $1 \mathrm{~mm}$ in skin biopsies from SCI Persons suffering with permanent denervation of thigh muscles from less of 1 to 8 years SCI The column of "Years from SCI" shows the number of years from SCI to enrolment for hbFES training.

\begin{tabular}{|c|c|c|c|c|c|c|c|}
\hline & & \multicolumn{2}{|c|}{ Interdigitation Index } & \multicolumn{4}{|c|}{ Number of papillae } \\
\hline & $\begin{array}{c}\text { Years } \\
\text { from } S C I\end{array}$ & Mean \pm SD & $\Delta \%$ & $\mathbf{p}$ & Mean \pm SD & $\Delta \%$ & $\mathbf{P}$ \\
\hline Group 1 & $0.8-3.9$ & $1.26 \pm 0.14$ & & & $5.34 \pm 1.31$ & & \\
\hline Group 2 & $4.1-8.0$ & $1.18 \pm 0.11$ & -22 & 0.029 & $5.54 \pm 2.98$ & +23 & 0.159 \\
\hline
\end{tabular}




\section{Discussion}

The skin is a sensory organ that protect the internal body from chemical, physical and biological insults. ${ }^{26,27}$ Different studies have shown that electrical stimulation has positive effects, specifically on wound closure. ${ }^{28-30}$ Based upon those evidence we were encouraged to study the effects of hbFES on the skin of patients with spinal cord injury, specifically in persons suffering with permanent denervation of the leg muscles as the result of conus and cauda equina complete lesion. ${ }^{1-8}$ Earlier, we demonstrated by sound functional, structural, ultrastructural and cellular approaches that 2-years hbFES produces significant improvements in muscle size and assisted contractile properties of the electro stimulated thigh muscles. ${ }^{1-13}$ We have indeed shown that denervated, degenerating muscles (DDM), of patients suffering with permanent disconnection of muscle fibers from the nervous system were rescued by two years of hbFES, using very large skin-contact electrodes and a new electrical stimulator designed in Vienna (Austria) ${ }^{2-8}$ and now commercially available. ${ }^{14}$ The use of those equipments and the related protocols of stimulation of DDM has been validated by the successful EU Program: RISE [Use of electrical stimulation to restore standing in paraplegics with long-term DDMs (QLG5-CT-200102191)]..$^{1-8}$ Thanks to the generous efforts of the Schuhfried Company based in Vienna, Austria the needed stimulators and large electrodes that recover DDM muscles are now commercially available. ${ }^{14} \mathrm{We}$ have previously demonstrated that the positive effects of hbFES are extended to the epidermal layer of the skin. ${ }^{15,16}$ The aim of the present report was to describe a different approach to hopefully confirm in incoming analyses the improvement of epidermis after two years of h-bFES. The previously studies evaluated the histological structure of skin and the extent of changes that occurred in the thickness of the epidermis of three human SCI persons before and after two years of continuous treatments of thigh muscles with hbFES by surface stimulation revealing that there was a significant $28 \%$ overall increase in the thickness of the epidermis in response to h-bFES. ${ }^{15}$ Then, with evaluation of epidermis area in cross sections of the skin and an unpaired statistical analysis the results were that the analysis of the grouped subjects showed a significant $30 \%$ increase after hbFES. ${ }^{16}$ Here we confirm, by further analyses in a larger sample of skin biopsies, that the clinical evidence of progressive skin atrophy and flattening in those series of RISE enrolled persons, i.e., suffering denervation atrophy of the legs from 1 to 8 years of SCI, negatively correlate also with the Interdigitation Index, i.e., with a sound biomarker of dermal papillae morphometry. ${ }^{31-34}$ Though the absolute value seems to present a low decrease (from 1.26 to 1.18 ), we stress that the Interdigitation Index varies from 1.3 in normal skin to 1.0 in fully flattened skin and that the standard Deviation of the present series of data is very low. Thus, we are confident that this parameter will provide conclusive evidence that the positive effects of hbFES will be supported by the incoming analyses in the series of biopsies harvested after two additional years of hbFES, but during which a subset of enrolled paraplegics performed hbFES managements with high compliance. Furthermore the incomming analyses are providing statistical evidence that the atrophy and flattening of the skin is related more to extent of SCI years than the difference in age of the SCI persons (Albertin et al., personal communication).

In conclusion, the Interdigitation Index is an additional sound quantitative structural biomarker of skin atrophy and flattening occurring in SCI, strongly supporting in a more numerous series of skin analyses our previous results of progressive decrease of epidermis thickness and area with extent of years from the SCI event. ${ }^{15,16}$ The result correlates with the much severe extent of atrophy of the permanently denervated thigh muscles, as determined at both macro and microscopic levels. ${ }^{1-8} \mathrm{We}$ are confident that the Interdigitation Index will provide sound evidence that the effects of hbFES, we previously reported on skeletal muscle and epidermis thickness, will be extended to the dermal layer of the skin, suggesting a coordinated negative effects of SCI extent on skeletal muscle and skin, and an improvement of both tissues after hbFES. ${ }^{1-3.15,17,19-24}$ Incoming analyses will be extended to basal lamina, collagene types, elastic fibers and skin annexes in the subcutaneous layer.

These results may be also relevant to other skin conditions, e.g., decubitus ulcer in SCI, metabolic disease late aging andwound healing. ${ }^{27-29,35-50}$

\section{List of acronyms}

DDM - denervated, degenerating muscles

FES - Functional Electrical Stimulation

hb FES - home-based FES

RISE Project - EU Program: RISE [Use of electrical stimulation to restore standing in paraplegics with longterm DDMs (QLG5-CT-2001-02191)]

SCI - Spinal Cord Injury

\section{Author's contributions}

Authors equally contributed to the manuscript.

\section{Acknowledgments and Funding}

The support of the European Regional Development Fund-Cross Border Cooperation Program SLOVAKIAAUSTRIA (Interreg- Iva) project 'Mobilität im Alter' MOBIL N_00033; Austrian Federal Ministry of Science and Research; Ludwig Boltzmann Society (Vienna) is gratefully acknowledged. Supported also by institutional funds of the Department of Neuroscience, Section of Human Anatomy, University of Padova, Italy; Interdepartmental Research Center of Myology, Department of Biomedical Sciences of the University of Padova, the IRCCS Fondazione Ospedale San Camillo, 
Venice, Italy. SZ thanks for support A\&C M-C Foundation for Translational Myology, Padova, Italy.

\section{Conflict of Interest}

The authors report no conflicts of interests.

\section{Ethical Publication Statement}

We confirm that we have read the Journal's position on issues involved in ethical publication and affirm that this report is consistent with those guidelines.

\section{Corresponding Author}

Barbara Ravara, Interdepartmental Reseach Centre of Myology, c/o Department of Biomedical Sciences, University of Padova, Italy.

E-mail: barbara.ravara@unipd.it

\section{E-mails of co-authors}

Helmut Kern: helmut@kern-reha.at Christian Hofer : christian.hofer@wienkav.at Diego Guidolin: diego.guidolin@unipd.it Andrea Porzionato: andrea.porzionato@unipd.it Raffaele De Caro: raffaele.decaro@unipd.it Giovanna Albertin: giovanna.albertin@unipd.it

\section{References}

1. Kern H, Hofer C, Löfler S, et al. Atrophy, ultrastructural disorders, severe atrophy and degeneration of denervated human muscle in SCI and Aging. Implications for their recovery by Functional Electrical Stimulation, updated 2017. Neurol Res 2017;39:660-6. doi: 10.1080/01616412.2017.1314906. Epub 2017 Apr 13.

2. Kern H, Carraro U, Adami N, et al. Home-based functional electrical stimulation rescues permanently denervated muscles in paraplegic patients with complete lower motor neuron lesion. Neurorehabil Neural Repair. 2010;24:709-721. DOI:10.1177/1545968310366129. Epub 2010 May 11.

3. Kern H, Carraro U, Adami N, et al. One year of home-based functional electrical stimulation (FES) in complete lower motor neuron paraplegia: recovery of tetanic contractility drives the structural improvements of denervated muscle. Neurol Res 2010;32:5-12. DOI:1 0.1189/184313209X385644

4. Fiorucci R, Piscioneri A. FES for large denervated muscles: comments of patients and practical demonstrations. Eur J Transl Myol 2013;23:162-4.

5. Kern H, Carraro U. Home-Based Functional Electrical Stimulation for Long-Term Denervated Human Muscle: History, Basics, Results and Perspectives of the Vienna Rehabilitation Strategy. Eur J Transl Myol 2014 Mar 27;24(1):3296. doi: 10.4081/ejtm.2014.3296. eCollection 2014 Mar 31

6. Kern H, Boncompagni S, Rossini K, et al. Longterm denervation in humans causes degeneration of both contractile and excitation contraction coupling apparatus, which is reversible by functional electrical stimulation (FES): A role for myofiber regeneration? J Neuropathol Exp Neurol. 2004;63:919-31.

7. Boncompagni S, Kern H, Rossini K, et al. Structural differentiation of skeletal muscle fibers in the absence of innervation in humans. Proc Natl Acad Sci USA 2007;104:19339-44. DOI: 10.1073/pnas.0709061104 PubMed PMID: 18042706; PubMed Central PMCID: PMC 2148291.2008;18:39-44.

8. Carraro U, Boncompagni S, Gobbo V, et al. Persistent muscle fiber regeneration in long term denervation. Past, present, future. Eur J Transl Myol 2015;25:77-92. DOI: 10.4081/ejtm.2015.4832

9. Carraro U, Kern H. Severely atrophic human muscle fibers with nuclear misplacement survive many years of permanent denervation. Eur J Transl Myol 2016;26:76-80. doi:10.4081/ejtm.2016.5894. eCollection 2016.

10. Gargiulo P, Reynisson PJ, Helgason B, et al. Muscle, tendons, and bone: structural changes during denervation and FES treatment. Neurol Res 2011;33:750-8. doi: 10.1179/1743132811Y .0000000007 .

11. Edmunds KJ, Gíslason MK, Arnadottir ID, et al. Quantitative computed tomography and image analysis for advanced muscle assessment. Eur $\mathbf{J}$ Transl Myol 2016 Jun 22;26:6015. DOI:10.4081/ejtm.2016.6015. eCollection 2016 Jun 13.

12. Carraro U, Edmunds KJ, Gargiulo P. 3D False Color Computed Tomography for Diagnosis and Follow-Up of Permanent Denervated Human Muscles Submitted to Home-Based Functional Electrical Stimulation. Eur J Transl Myol 2015;25:5133. doi: 10.4081/ejtm.2015.5133. eCollection 2015 Mar 11. Review.

13. Ortolan P, Zanato R, Coran A, et al. Role of Radiologic Imaging in Genetic and Acquired Neuromuscular Disorders. Eur J Transl Myol 2015;25:5014. doi: 10.4081/ejtm.2015.5014. eCollection 2015 Mar 11. Review.

14. Available: https://www.schuhfried.com/umbraco /Surface/AuthenticationSurface/Login?returnUrl= \%2Fportal

15. Albertin G, Hofer C, Zampieri S, et al. In complete SCI patients, long-term functional electrical stimulation of permanent denervated muscles increases epidermis thickness. Neurol Res 2018 Feb 15:1-6. doi: 10.1080/01616412.2018.1436877. [Epub ahead of print].

16. Albertin G, Kern H, Hofer C, et al. Two years of Functional Electrical Stimulation by large surface electrodes for denervated muscles improve skin epidermis in SCI. Eur J Transl Myol 2018;28:7373. 
doi: 10.4081/ejtm.2018.7373. eCollection 2018 Jan 12.

17. Timar F, Soos G, Szende B, Horvath A. Interdigitation index - a parameter for differentiating between young and older skin specimen. Skin Res Technol. 2000;6:17-20.

18. Available at http://rsb.info.nih.gov/ij/

19. Porzionato A, Macchi V, Guidolin D, et al. Histopathology of carotid body in heroin addiction. Possible chemosensitive impairment. Histopathology. 2005;46:296-306.

20. Kern H, Gargiulo P, Pond A, et al. To reverse atrophy of human muscles in complete SCI lower motor neuron denervation by home-based Functional Electrical Stimulation, in Muscle Atrophy, ed J. Xiao. Advances in Experimental Medicine and Biology 2019;1088, https://doi.org/10.1007/978-981-13-1435-

3_252019. https://doi.org/10.1007/978-981-131435-3_27.

21. Carraro U, Gava K, Baba A, et al. Fighting muscle weakness in advanced aging by take-home strategies: Safe anti-aging full-body in-bed gym and functional electrical stimulation (FES) for mobility compromised elderly people. Biol Eng Me 2018;1. $1-4$.

22. Carraro U, Gava K, Baba A, et al. To contrast and reverse skeletal muscle atrophy by Full-Body InBed Gym, a mandatory life-style for older olds and borderline mobility impaired persons, in Muscle Atrophy, ed J. Xiao. Adv Exp Med Biol 2019;1088, https://doi.org/10.1007/978-981-13-1435-3_25.

23 Mosole S, Zampieri S, Furlan S, et al. Effects of Electrical Stimulation on Skeletal Muscle of Old Sedentary People. Gerontol Geriatr Med 2018;4:2333721418768998. doi: 10.1177/2333721418768998. eCollection 2018 Jan-Dec.

24. Edmunds K, Gíslason M, Sigurdsson $\mathrm{S}$, et al. Advanced quantitative methods in correlating sarcopenic muscle degeneration with lower extremity function biometrics and comorbidities. PLoS One 2018;13(3):e0193241. doi: 10.1371/journal.pone.0193241. eCollection 2018.

25. Gargiulo P, Gislason, MK, Edmunds KJ, et al. CTBased Bone and Muscle Assessment in Normal and Pathological Conditions. Encyclopedia of Biomedical Engineering, 2019; Vol. 2:119-34. http://doi.org.10.1016/B978-0-801238-3.99920-3.

26. Bloom W, Fawcett DW. A textbook of histology, 12th ed., Chapman and Hall, 1994.

27. Lauria G, Lombardi R, Camozzi F, Devigili G. Skin biopsy for the diagnosis of peripheral neuropathy. Histopathology. 2009; 54:273-285. DOI: 10.1111/j.1365-2559.2008.03096.x. Epub 2008 Jul 15.

28. Hunckler J, de Mel A. A current affair: electrotherapy in wound healing. J Multidiscip
Healthc 2017:10:179-94. DOI: 10.2147/JMDH.S 127207. eCollection 2017.

29. Zhao M, Bai H, Wang E, et al. Electrical stimulation directly induces pre-angiogenic responses in vascular endothelial cells by signaling through VEGF receptors. J Cell Sci 2004;117(Pt 3):397405. Epub 2003 Dec 16 DOI: 10.1242/jcs.00868.

30. Bayat M, Asgari-Moghadam Z, Maroufi M, et al. Experimental wound healing using microamperage electrical stimulation in rabbits. J Rehabil Res Dev 2006;43:219-26.

31. Wang YN, Lee K, Ledoux WR. Histomorphological evaluation of diabetic and nondiabetic plantar soft tissue. Foot Ankle Int. 2011 Aug;32:802-10.

32. Sauermann K, Clemann S, Jaspers S, et al. Age related changes of human skin investigated with histometric measurements by confocal laser scanning microscopy in vivo. Skin Res Technol. 2002 Feb;8:52-6.

33. Liao YH, Kuo WC, Chou SY, et al. Quantitative analysis of intrinsic skin aging in dermal papillae by in vivo harmonic generation microscopy. Biomed Opt Express. 2014 Aug 28;5(9):3266-79. doi: 10.1364/BOE.5.003266. eCollection 2014 Sep 1.

34. Morrison T, Jones S, Causby RS, Thoirs K. Can ultrasound measures of intrinsic foot muscles and plantar soft tissues predict future diabetes-related foot disease? A systematic review. PLoS One. 2018 Jun 15;13(6):e0199055. doi: 10.1371/journal.pone. 0199055. eCollection 2018.

35. Singer AJ, Clark RA. Cutaneous wound healing. N Engl J Med 1999;341:738-746. Review.

36. Gosain A, DiPietro LA. Aging and wound healing. World J Surg 2004;28:321-6. Epub 2004 Feb 17. Review.

37. Boyer B, Kern P, Fourtanier A, et al. Agedependent variations of the biosyntheses of fibronectin and fibrous collagens in mouse skin. Exp Gerontol1991;26:375-83.

38. Zhao M, Song $\mathrm{B}, \mathrm{Pu} \mathrm{J}$, et al. Electrical signals control wound healing through phosphatidylinositol-3-OH kinase-gamma and PTEN. Nature 2006;442:457-60.

39. Norman RA, Henderson JN. Aging: an overview. Dermatologic Therapy 2003;16:181-185 DOI: 10.1046/j.1529-8019.2003.01627.x.

40. El-Domyati M, Attia S, Saleh F, et al. Intrinsic aging vs. photoaging: a comparative histopathological, immunohistochemical, and ultrastructural study of skin. Exp Dermatol. 2002;11:398-405. PubMed PMID: 12366692 DOI: 10.1034/j.1600-0625.2002.110502.x.

41. Barberi, L, Scicchitano BM, Musaro A. Molecular and cellular mechanisms of muscle aging and sarcopenia and effects of electrical stimulation in seniors. 2015 Eur J Transl Myol 2009;25:231-6. 
doi: 10.4081/ejtm.2015.5227. eCollection 2015 Aug 24. Review.

42. Pigna E, Berardi E, Aulino P, et al. Aerobic Exercise and Pharmacological Treatments Counteract Cachexia by Modulating Autophagy in Colon Cancer. Sci Rep 2016 May 31;6:26991. doi: 10.1038/srep26991.

43. Coletti D. Chemotherapy-induced muscle wasting: an update. Eur J Transl Myol 2018 Jun 4;28(2):7587. doi: 10.4081/ejtm.2018.7587. eCollection 2018 Apr 24.

44. Gava P, Kern H, Carraro U. Age-associated power decline from running, jumping, and throwing male masters world records. Exp Aging Res 2015;41:115-35. doi: 10.1080/0361073X.2015.1001648.

45. Carraro U, Gava K, Musumeci A, et al. Safe Antiaging Full-Body In-Bed Gym and FES for Lazy Persons: Home In-Bed Exercises for Fighting Muscle Weakness in Advanced Age, in Rehabilitation Medicine for Elderly Patients, eds S. Masiero and U. Carraro (Springer-Nature Book), 2018, pag. 43-52. ISBN 978-3-319-57405-9 ISBN 978-3-319-57406-6 (eBook) 30.
46. Mitchell WK, Williams J, Atherton $\mathrm{P}$, et al. Sarcopenia, dynapenia, and the impact of advancing age on human skeletal muscle size and strength; a quantitative review. Front Physiol 2012 Jul 11;3:260. doi: 10.3389/fphys.2012.00260. eCollection 2012.

47. Krenn M, Haller M, Bijak, M, et al. Safe neuromuscular electrical stimulator designed for the elderly. Artif Organs 2011;35:253-6.

48. Hamar D. Universal linear motor driven Leg Press Dynamometer and concept of Serial Stretch Loading. Eur J Transl Myol 2015;25:215-9.

49. Cvecka J, Tirpakova V, Sedliak M, et al. Physical activity in elderly. Eur. J. Transl. Myol.2015; 25:249-52.

50. Sarabon N, Löfler S, Hosszu G, Hofer Ch. Mobility test protocols for the elderly: a methodological note. Eur J Transl Myol 2015;25:253-6.

Received for publication: 05/11/2018

Accepted for publication: 12/11/2018 